dependence of the scattering intensity is of particular interest in that, for certain parameter ranges (such as the maxon and roton regions) it consists of two principal features: a sharp peak superimposed upon a broader background (Fig. 4). It is evident that the sharp peak disappears above the transition temperature, $T_{\lambda}$, at which the superfluid turns normal.

Considerable debate centred on the physical significance of the relative intensity of the two peaks in Fig. 4. A widely accepted perception, clearly articulated by A. Griffin (University of Toronto), is that the phonons on one hand, and the rotons and maxons on the other, are inherently different kinds of excitation quite to the contrary of what one might assume from the continuous character of the dispersion curve in Fig. 1. Phonons, being density fluctuations of the whole fluid, are detected by neutron scattering both above and below $T_{\lambda}$. Rotons and maxons, on the other hand, are more localized 'single-particle' excitations of some (unspecified) kind, and are probably characterized by an entirely distinct dispersion curve above $T_{\lambda}$ at which

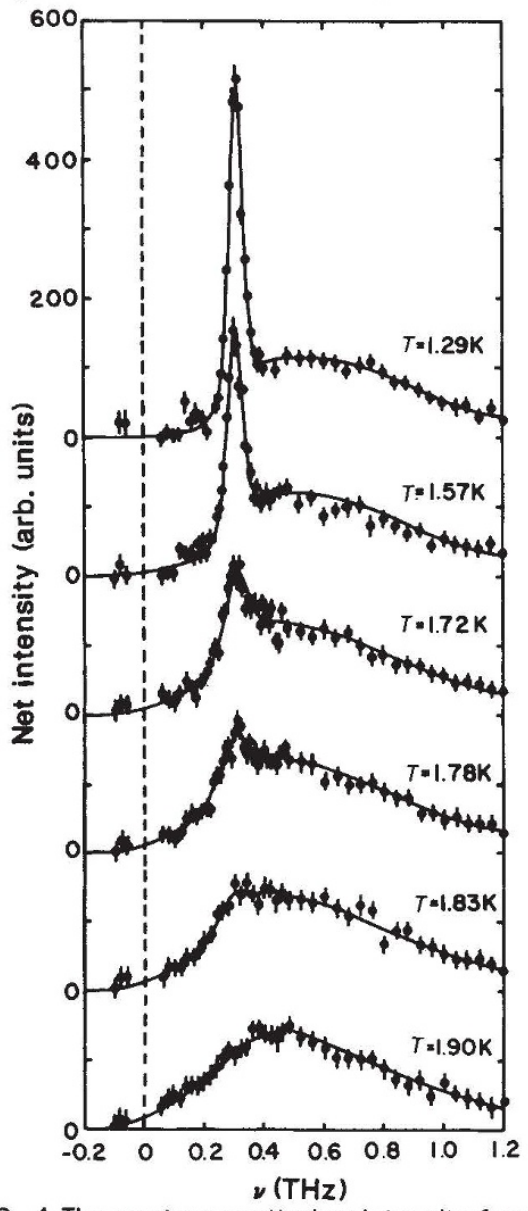

FIG. 4 The neutron scattering intensity from superfluid helium at the maxon, plotted as a function of energy (divided by Planck's constant h) for several temperatures. For the ambient pressure of 20 bar, the superfluid transition temperature $T_{\lambda}=1.93 \mathrm{~K}$. It is evident that the sharp peak disappears at $T_{\lambda}$. (From ref. 9.) temperature they cannot, unfortunately, be detected by neutron scattering. Below $T_{\lambda}$, the existence of a small fraction $n_{0}$ of the helium atoms occupying the zeromomentum 'Bose-condensed' state has profound theoretical consequences. In particular, it causes the phonon and roton/maxon branches of the dispersion curve to combine, through a quantummechanical process known as hybridization, thereby forming the single dispersion curve observed in the experiments. Correspondingly, the maxon/roton excitations then become detectable by neutron scattering (the sharp peak evident in Fig. 4) with a weight that is proportional to the fraction $n_{0}$.

This argument depends, of course, on the existence of a non-zero condensate fraction (which should be carefully distinguished from the superfluid fraction, representing the proportion of the fluid which displays superfluid properties and which approaches 100 per cent below $1 \mathrm{~K}$ ). Fortunately, $n_{0}$ itself can also be measured by inelastic neutron scattering, albeit in collisions with a much higher transfer of momentum. P. E. Sokol (Pennsylvania State University) was thereby able to derive a value of $n_{0} \sim 0.10$ as $T \rightarrow 0$, agreeing within experimental error with earlier neutron results by Svensson and co-workers ${ }^{4}$ based on a rather different approach. This is of course very much a finite value and it is also consistent with recent theoretical predictions.

Given that rotons crop up in all facets of superfluid helium-4 research - lightscattering studies, diffusion of helium-3 through the superfluid, and the crystallization of solid helium-4, for example, all discussed at the meeting - it is remarkable that so little is known of their physical character. But then, after 50 years of study, superfluid helium-4 remains in many ways much more mysterious than its younger relatives superfluid helium- 3 and superconductivity. The prospect held out at the meeting is that we should soon come to grips with what makes it so much more complex.

P. V. E. McClintock is in the Department of Physics, Lancaster University, Lancaster LA1 $4 Y B, U K$.

1. Landau, L. D. J. Phys. U.S.S.R. 5, 71 (1941); 11, 91 (1947).

2. Feynman, R. P. in Progress in Low Temperature Physics Vol. 1 (ed. Gorter, C. J.) Ch. 2 (North-Holland, Amsterdam, 1955)

3. Jones, C. A. \& Roberts, P. H. J. Phys. A15, 2599 (1982)

4. Sears, V. F., Svensson, E. C., Martel, P. \& Woods, A. D. B. Shys. Rev. Lett. 49, 279 (1982)
Phars, F. Svensson, E. C., Marte

5. Svensson, E. C. in Elementary Excitations in Quantum Fluids (eds Ohbayashi, K. \& Watabe, M.) 59-- 95 (Springer, Berlin, 1989)

6. Donnelly, R. J., Donnelly, J. A. \& Hills, R. N. J. Low Temp. Phys. 44, 471 (1981).

7. Samuels, D. C. \& Donnelly, R. J. Phys. Rev. Lett. 65, 187-190 (1990).

8. Forbes, A. C. \& Wyatt, A. F. G. Phys. Rev, Lett. 64, 13931396 (1990).

9. Talbot, E. F., Glyde, H. R., Stirling, W. G. \& Svensson, E. C. Phys. Rev. B38, 11229 (1990)

\section{Deep digestion}

Most land animals use the nitrogen in their food proteins very inefficiently. They excrete it as urea or uric acid, making no attempt to burn these waste products usefully. Yet urea and uric acid are quite feasible fuels. As manufacturers of high explosives know, an oxidative decomposition which releases free nitrogen gas also releases a lot of energy. Why don't animals exploit this energy?

The reason, says Daedalus, is that nitrogen is almost insoluble in water. Carbon dioxide, our main gaseous waste product, is soluble enough to be transported round the body in solution. But a creature that generated nitrogen internally would find itself in trouble. It would swell up with bubbles of undissolved gas, and might even burst.

Divers risk a similar fate. Under the high pressure of the depths, the nitrogen in the air they breathe dissolves in their body fluids. When they come back to normal pressures it comes out again, and may form bubbles in the blood or synovial fluid. This is the painful and dangerous diving syndrome, 'the bends'.

So, says Daedalus, the ultimate metabolic efficiency should be shown by deepsea creatures, living under pressures high enough for nitrogen to be easily soluble in water. Freed from solubility problems, they could metabolize proteins and other nitrogenous foods completely to nitrogen, perhaps feasting on the steady fall of nitrogenous wastes from the low-pressure creatures far above them.

DREADCO's biologists are now studying deep-sea lantern-fish, worms and sponges in special pressurized aquaria and culture vessels, looking for novel enzymes that metabolize urea and other nitrogenous wastes. If they find them, they will look for traces of them in land creatures too. A small amount of nitrogen metabolism, not enough to cause the bends but giving a little added efficiency while slightly shrinking the excretion load, could well have evolved in many species. Selective breeding, or even genetic engineering, could develop it further.

At first Daedalus dreamt of breeding a special non-excreting urban dog, which metabolized its food all the way down to nitrogen gas; but he soon realized that at atmospheric pressure it would rapidly succumb to the bends. He then recalled his previous scheme for a high pressure hen house or turkey coop, to revive the flight instincts of these sedate birds. So he now plans to develop a breed of non-excreting high-efficiency chickens, turning nearly all their food into meat and eggs with minimum waste and mess. Their special coop will be pressurized with oxygen and argon, and tended by farm labourers in diving gear.

David Jones 